\title{
Biological indicators and their clinical significance in persons exposed to mineral fibres: report of a workshop held in Japan, 24-25 November 1991
}

A key ingredient in the development of rational programmes for the prevention of fibre related health effects is that the indicators of health effects used to monitor the workforce or to detect early effects be related in a meaningful way to the health outcomes that the programmes are designed to prevent.

In November 1991, the University of Occupational and Environmental Health and the Scientific Committee on Mineral fibres of the International Commission on Occupational Health $(\mathrm{ICOH})$ organised a workshop on this subject. This workshop was held in conjunction with the international symposium on the health effects of low exposure to fibrous minerals at the University of Occupational Health and Environmental Health, Kitakyushu, Japan, 24 and 25 November 1991.

The workshop and some of the associated papers form part of the proceedings of that symposium, which is to be published in: Gibbs G W, Dunnigan $\mathrm{J}$, Kido $\mathrm{M}$, Higashi $\mathrm{T}$, eds. Health risks from exposure to mineral fibres: an international perspective. Concord, Ontario, Canada: Captus Press, 1993.

The workshop brought together scientists with special expertise in this area who were provided with questions to be discussed in advance of the workshops but specific review papers were not prepared.

These summaries by the rapporteurs reflect the deliberations of the two working groups (workshops 1 and 2) who, after a plenary session, met to consider different aspects of the subject.

The opinions expressed in relation to specific questions were based on materials presented at the workshop (see list of presentations at the end of the report), personal knowledge, experience, and expertise of members of the working groups.

Introduction prepared and manuscript submitted by G W Gibbs, Chairman ICOH Scientific Committee on Mineral Fibres

Safety Health Environment International Box 27, Site 17, RR2 Winterburn, Alberta, Canada TOE 2 NO

\section{Workshop 1}

MEMBERS OF WORKING GROUP

M Becklake (rapporteur, Department of Epidemiology and Biostatistics, McGill University 1020 Pine Avenue West, Montreal, Quebec, H3A 1A2 Canada), J Bignon (chair), P Brochard, G Chiappino, A Cavalleri, A Ward Gardner, KG Hering, Y Hosoda, D Liddell, M Tanaka, W Gardner.

COMMENT ON BIOLOGICAL INDICATORS

The group noted that the term "biological indicator" can be used in more than one way, these are: (1) to indicate exposure or retention of the putative agent and/or (2) biological response to this exposure.

The term was used in both ways by the working group and several presentations at the workshop dealt with both issues.

\section{QUESTIONS AND DISCUSSION}

Working group 1 considered two questions formulated to correspond to the objectives of the workshop.

\section{QUESTION 1}

Are clinical indicators including radiological changes predictive of:

(a) life shortening and/or death with particular reference to pneumoconiosis, lung cancer, mesothelioma, and other respiratory conditions, and/or (b) respiratory impairments?

Radiological changes are widely used in the assessment of asbestos related pulmonary disease but few studies have directly addressed the question of the value of radiological changes as predictors of outcome. Apparently parenchymal radiological changes have been shown to predict mortality from several causes, including lung cancer. ${ }^{1}$

Another clinical concern in the 1990 s is the relevance to outcome of pleural radiographic changes 
with minimal or no associated parenchymal change, now a common form of clinical presentation. Three reports pertinent to this topic were presented at the workshop.

One was based on the study of 429 deaths in a cohort of chrysotile miners and millers of Quebec. Although only preliminary, analysis of the mortality up to 1986 confirmed earlier findings ${ }^{2}$ and indicates that specific radiological and clinical signs and respiratory symptoms are strongly associated with deaths attributed to certain causes and with total mortality.

The second study from Japan (Hosoda, this workshop) based on a 20 year cohort study with a few deaths suggests that local pleural changes, excluding costophrenic angle obliteration, were not significantly associated with higher overall mortality nor with a higher risk of lung cancer.

In the third study involving 173 workers exposed to asbestos who underwent bronchoalveolar lavage (BAL), pleural plaques were shown to be related to the internal lung burden as measured by asbestos bodies in BAL fluid (Chiappino et al, this workshop). Thus when associated with a low internal burden, pleural plaques seem to be markers of exposure; when associated with a high internal burden, pleural plaques seem to be markers of a biological response that will result in asbestosis.

Thus there is evidence from these three reports, two of which are preliminary, that radiological parenchymal changes and possibly pleural changes may predict outcome, including mortality. It must, however, be emphasised that the inference that pleural changes predict mortality outcomes runs counter to the findings in the study by Hughes and Weill, ${ }^{1}$ in which certain degrees of parenchymal change predicted mortality but pleural changes did not. Rationalisation will be required when the full reports of the findings presented to the workshop are available.

The second part of the question deals with radiological changes as predictors of respiratory impairment. Evidence from the cross sectional workforce based studies reviewed (Becklake, this workshop) indicates that: (1) benign pleural manifestations of exposure to fibrous minerals are associated with reduction in the level of spirometric measurements, including FVC and $\mathrm{FEV}_{1}$, independently of any associated parenchymal change; (2) the reduction in the level of these spirometric values seems to be proportional to the extent to which the pleural surfaces on both sides are involved; (3) blunting of the costophrenic angle, implying a more diffuse involvement of the pleura, seems to be associated with greater deficits in spirometric values than chest wall abnormality.

Information in two other reports (Chiappino, Hosoda, this workshop) also indicated that certain radiological pleural changes were associated with deficits in ventilatory function.

It was noted that the information on which these conclusions were reached was based on population (epidemiological) studies and that in so far as the individual case with radiological pleural abnormality is concerned, impairment could range from undetectable to considerable.

\section{QUESTION 2}

What is the clinical and prognostic significance of observations based on newer methods of study?

One report (Hering, this workshop) analysed the additional information provided by high resolution computed tomography (HRCT) in about 400 cases of asbestos exposed workers in whom diffuse pleural thickening (ILO $1980>2 \mathrm{a}$ ) was recorded on the posterioanterior chest film. The analysis led to the conclusions: (1) HRCT identified parenchymal changes underlying the pleural thickening in many cases; (2) pleural thickening was often seen in the neighbourhood of a fat pad; (3) besides localised parietal pleural plaques and diffuse pleural thickening, other features that should be noted include flat, parietal plaques that may be confluent, and regional or local fibrosis of the visceral pleura; (4) sonography, magnetic resonance imaging, and digital luminescence radiography added little information although they may be useful in the investigation of particular cases.

Studies are now being undertaken to determine whether HRCT has a role in the examination of workers at high risk, despite the radiation dose associated with the procedure. At issue is how to reduce the number of cuts (and radiation dose), while minimising the loss of sensitivity.

Another recommendation is that pleural thickening $>2 \mathrm{a}$ according to the ILO 1980 scale ( $>$ $3 \mathrm{~mm}$ on the posterioarterior chest radiograph) should be recorded by readers even in the absence of blunting of the costophrenic angle.

The concentration of aminoterminal peptide of procollagen III (P III NP) in serum is thought to be an index of early active fibrosis and correlates well with the deposition of collagen III. Evidence in support of this view was provided by a radioimmunoassay of serum P III NP in two separate studies of asbestos cement workers (Cavalleri, this workshop). P III NP was related to exposure level, and was also associated with individual responsiveness and with impairment of lung function, but not with the presence of established fibrosis.

The working group concluded that P III NP measurements in serum could be used to consider research questions related to biological monitoring strategy, but should not be used as a diagnostic tool. Longitudinal studies are needed to confirm its 
role as an indicator of fibrogenesis and if this was shown to be the case, the measurement has potential as a screening test.

Clinical procedures for diagnosis of pleural mesothelioma available at present including computed tomography scan, HRCT, or thoracoscopy, confirm the diagnosis only at an advanced stage of the disease. There is a need to improve diagnostic methods and to discover new markers.

This issue is of major concern, as new therapeutic approaches (local immunotherapy, gene therapy etc) are being developed and the response to treatment might be improved if it is applied at an earlier stage of the disease. ${ }^{3}$

GENERAL CONCLUSIONS: WORKSHOP 1

(1) The working group noted that, in current clinical practice, fibrosis consequent on exposure to asbestos, and manifest as radiographic change, is seen as often in the pleura as in the lung parenchyma. Because the prevalence of all but slight profusion of small opacities is generally falling, pleural radiographic changes, with minimal or no parenchymal changes, are now the commonest form of clinical presentation. This contrasts with the carcinogenic effects found in cohort studies of asbestos exposed workers in which the number of malignant mesotheliomas of the pleura has been less than half the number of excess lung cancers. (2) Therefore, there is urgent need to establish the relative clinical and/or predictive significance of the various pleural and parenchymal changes, occurring separately and in conjunction, and taking into account type and site of changes, and their severity. (3) Another pressing need is for a review of the current ILO International Classification of Radiographs of Pneumoconioses, which dates from 1980; this should take account of newer pleural appearances. (4) Certain new biological indicators seem exciting, but carefully designed studies are needed to evaluate their clinical significance, with a view to exploitation and further development.

\section{Workshop 2}

MEMBERS OF WORKING GROUP

B Case (rapporteur, Department of Pathology, McGill University, 3775 University Street, Montreal, Quebec, Canada), CE Rossiter (chair), M Saux, K Browne, M Huuskonen, K Takahashi, M Kawanami

\section{QUESTIONS AND DISCUSSION}

QUESTION 1

Are indicators of exposure such as ferruginous bodies predictive of later adverse health effects and/or prognosis?
Indicators of exposure to mineral fibres include markers of asbestos internal dose such as:

(1) Ferruginous bodies in sputum; (2) ferruginous bodies in bronchoalveolar lavage (BAL) (alveolar "burden"); (3) ferruginous bodies recovered from lung parenchyma obtained during lung surgery or at autopsy (lung "burden").

Lung parenchyma and BAL fluid also provide a source of tissues for analytical transmission electron microscopy (ATEM) and scanning electron microscopy (SEM) measurements of specific mineral fibre types (including chrysotile, amphiboles, manmade mineral fibres, and other inorganic fibres and mineral fragments) and quantities. Results are usually expressed as million fibres/g dry lung or fibres/ $\mu \mathrm{g}$ dry lung.

New information is becoming available on nonspecific markers of biological effects. Data reported from Cavalleri's laboratory (Cavalleri, this workshop and noted in workshop 1) suggest that serum peptides associated with the synthesis of type III collagen are related in a dose dependent fashion to exposure in an asbestos cement factory, down to as low as 0.2 fibres $/ \mathrm{ml}$ air. In earlier work in Chiappino's laboratory, however, these markers of collagen synthesis were not useful in patients with active, ongoing asbestos related fibrosis. Further work to explain this paradox is necessary.

The working group noted that it was important to know the degree to which biological indicators were representative of exposures before questions of disease prediction could be properly considered.

Sputum-There was consensus that the finding of ferruginous bodies in general populations is a rare event $(0.3 \%)$, and that use of sputum ferruginous bodies is too insensitive for use in individual exposure assessment. The finding of even one "typical" asbestos body in sputum is, however, suggestive of occupational exposure to asbestos.

Results of cross sectional epidemiological studies with sputum ferruginous bodies have varied from $0 \%(0 / 43)$ in one group of custodial workers and $6 \%$ to $18 \%$ in selected environmentally exposed groups to as high as $70 \%$ in one group of vermiculite miners heavily exposed to asbestiform tremolite. The last finding is unusual; in most occupational groups the test is less sensitive.

Sputum adequacy needs to be assessed by alveolar macrophage counts. Absence of sputum ferruginous bodies does not denote definite absence of exposure, as technical factors such as sputum productivity and confounding factors such as cigarette smoking and age intervene in unquantified ways. ATEM has rarely been used in the assessment of sputum fibre content, and the complexity of the technique makes it unlikely to be routinely productive. One study of workers exposed to amosite did, however, show that whereas ferrugi- 
nous bodies were not detectable with conventional chemical digestion and light microscopy, examination by ATEM revealed amosite fibres in sputum from most subjects.

Ferruginous body concentrations derived from $B A L$ fluid-These concentrations expressed per $\mathrm{ml}$ of recovered lavage fluid, are of increasing importance in exposure assessment. Separate reports from two laboratories have indicated with $95 \%$ confidence that BAL fluid counts exceeding one ferruginous body per $\mathrm{ml}$ recovered BAL fluid are related to lung content of ferruginous bodies in excess of $1000 / \mathrm{g}$ dry lung, and as such denote probable occupational exposure. This statement is limited to the specific techniques used and working populations assessed in the two laboratories. Data suggest that further work in other laboratories and on other populations may confirm this level as a "sentinel" value.

Measurement of fibres in BAL fluid by ATEM can be sensitive even when ferruginous bodies seem to be absent from BAL fluid with conventional light microscopical techniques. The working group suggested extension of existing BAL protocols to improve sensitivity and specificity, although realising that current data have limited clinical applicability in the case of the individual patient with putative exposure to asbestos.

Lung Parenchymal Ferruginous Bodies and Lung ATEM Parenchymal Fibres-There is an enormous body of work, dating as far back as the recognition of asbestosis itself, on lung parenchymal ferruginous bodies and lung parenchymal fibres.

\section{CONSENSUS STATEMENT-WORKSHOP 2}

(1) Formal interlaboratory exchange programmes are needed to standardise the techniques for the quantification of ferruginous bodies in lung tissue.

(2) There is a pressing need for interlaboratory work with ATEM techniques for the quantitative identification of inorganic fibres of all types.

(3) More studies such as recent work by McDonald $e t \mathrm{al}^{+}$are needed to aim specifically at the assessment of human exposures to manmade mineral fibres and other non-asbestos fibres.

(4) Methods need to be developed for the isolation of organic fibres, as current techniques remove these structures through tissue processing steps such as low temperature ashing and chemical digestion. Techniques such as those for the in situ assessment of non-fibrous particles should be developed for similar evaluations of fibres.

(5) The finding of a lung parenchymal content of greater than 1000 ferruginous bodies/g dry lung is highly suggestive of occupational exposure to asbestos, as long as the value is obtained with stan- dard methods in a laboratory with experience in the application of the technique. This sentinel value has been ascertained in an era of high level occupational exposure to asbestos and a lower value may prove to be a better cutoff point. It is especially important to relate lung parenchymal values to lung alveolar burden expressed as ferruginous bodies recovered per $\mathrm{ml}$ BAL fluid. Interpretation of lung parenchymal ferruginous body values under $1000 / \mathrm{g}$ dry lung is presently problematic, so that the measurement is highly specific but of uncertain sensitivity for the individual "negative result". A result from an individual lung parenchymal analysis above 1000 ferruginous body/g dry lung has meaning, but a result below 1000 denotes a lack of interpretable information.

(6) Further investigations of the relevance of lung internal dose markers to pleural plaques, other pleural changes, pleural lymphatic drainage, and pleural pathophysiology are needed. Important work by Sebastien et al ${ }^{5}$ suggesting that pleural fibre content is principally composed of short chrysotile fibres lacking any relation to lung parenchymal fibre content should be replicated and extended. Animal and human studies must be developed to determine once and for all how or whether inhaled minerals reach and are concentrated in the parietal pleura.

(7) There is a great need for pleural tissue burden studies in case-control comparisons for pleural diseases including malignant mesothelioma.

(8) In cross sectional and case-control studies the laboratory performing the analyses for ferruginous bodies or TEM fibres should obtain and apply control values from comparable unexposed populations. For example, Brochard and colleagues found differences between groups of French mesothelioma cases in areas of high fibre pollution due to shipbuilding (Nantes) $v$ those from an area with little direct asbestos related industry (Paris): findings of high concentrations of ferruginous bodies or TEM fibres in lungs have different meanings in different locations.

(9) The working group recommends the use of controls matched to the degree possible, for age, sex, and particularly time and place of necropsy or surgical resection and pathological processing. The last control process can help to avoid or to identify contamination of lung and pleural samples with exogenous materials, including asbestos, manmade mineral fibres, talc etc. This is important in the assessment of populations with low exposure, such as children. The findings by Case $e a^{6}$ that ATEM preparations of children's lungs were uniformly contaminated by short crocidolite fibres derived from one brand of polycarbonate filter is a cautionary instance. Effects of tissue processing techniques in the pathology room and in the 
research laboratory must be better defined, especially for more soluble fibres such as chrysotile and glass fibre.

(10) There is a continuing need to put the findings of studies of lung fibre content in proper epidemiological context. This has in the past been rendered difficult, partly because much of the work in the field has come from laboratories without adequate epidemiological input. Complexities such as those introduced by the technical factors involved in lung digestion and ashing studies and physiological and metabolic differences in the lung reaction to different fibre types must be taken into account.

(11) Interdisciplinary cooperation, particularly between pathologists, mineralogists, epidemiologists, and biostatisticians should be encouraged. Formation of working groups such as those assembled for the recent CIIT Symposium on manmade mineral fibres and the recent consensus report (HEI-AR report; asbestos in public and commercial buildings: a literature review and synthesis of current knowledge, 1991) are exemplary of a positive approach. This is one way in which the controversial issues (see later) can be considered. Multidisciplinary meetings should include representatives of diverging scientific points of view so that studies can be designed to reconcile these differences.

(12) If all of these precautions are taken, lung internal dose in a large series of studies of lung fibre content can be interpreted. These include general population exposures in many countries and in many periods, exposures in the neighbourhood of point sources such as open pit mines, shipyards, and asbestos cement factories, and domestic (household) exposures of the families of fibre mining, milling, manufacturing and application workers. The general neighbourhood and domestic groupings can be reliably separated as populations. Placing individual subjects within one of these categories on the basis of such data is more difficult, as there is overlap of test results.

(13) There was little discussion of the role of exposure indicators as predictors of later adverse health effects and prognosis. A growing body of published data concerning mesothelioma exists. These studies are generally consistent in suggesting that most subjects with asbestos related mesothelioma have ferruginous body counts in excess of $1000 / g$ dry lung, and an excess of amphibole asbestos fibres in lung tissue relative to matched controls. Variation in the results seems to reflect local differences such as predominent industries, point sources, and prevalence of either commercial or contaminant amphiboles.

(14) For the individual exposed subject, any given value of lung content of ferruginous bodies, whether by light microscopy or by ATEM for specific fibre type has no predictive value for the subsequent development of mesothelioma or other adverse health effect.

\section{Issues}

There is a need to challenge assumptions in a field in which much of the published data are based on small numbers of observations. Controversial areas identified by the group included:

(1) The apparent lack of effect of chrysotile on the development of mesothelioma; a finding based partly on epidemiological studies but also on studies of lung fibre content.

(2) The significance, if any, of the finding of cleavage fragments of tremolite in lung tissue.

(3) The importance of the length and diameter of fibres recovered from lung tissue, with a need for confirmation of animal experiments emphasising an almost exclusive role in pathogenesis for long (greater than 5 or greater than $8 \mu \mathrm{m}$ length) and thin (less than $0.5 \mu \mathrm{m}$ diameter) fibres. The group noted that the importance of these issues is underlined in preliminary findings on the relative frequency of long ( $>5 \mu \mathrm{m}$ ) chrysotile fibres and short $(<5 \mu \mathrm{m})$ tremolite fragments in the lung tissue of American children. It was pointed out that these findings, being several orders of magnitude lower than those found in adults, are not inconsistent with the possibility of contamination; certainly the results to date do not indicate specific cause for concern for any fibre type.

(4) Observations of diverging fibrotic response for similar dose, both in lung parenchyma and pleura, must be explained. These have been assumed to be the result of individual differences in susceptibility, but this has not been proved. Internal dose study coupled with the development of indicators of susceptibility would be useful.

\section{MONITORING AND SURVEILLANCE}

Although the members of the working group agreed that monitoring and surveillance of workers is an important subject, they did not consider the wide range of issues involved. One comprehensive approach to monitoring and surveillance is that used in Finland. This approach is fully outlined in the symposium paper by Huuskonen et al published in the conference proceedings (the Finnish action program against the health risks of asbestos).

PRESENTED PAPERS RELEVANT TO WORKSHOP 1

Hering KG et al. What can be measured by $x$ rays/CRT/ultrasound and the newer techniques?

Brochard P. Morbidity data of permanent users of buildings with asbestos containing materials. 
Becklake $\mathrm{M}$ et al. Asbestos related pleural abnormality: clinical significance and associated impairment.

Hosoda $\mathrm{Y}$ et al. A 20 year follow-up of asbestos insulation workers of radiographic normal lungs with and without pleural plaques.

Liddell FDK. Radiologic changes in chrysotile workers.

Bignon J. Fifteen year follow-up of indoor pollution by asbestos fibers in the buildings of Paris University.

Cavalleri A. Serum levels of amino-terminal peptide of procollagen III in monitoring low exposure to asbestos fiber.

Chiappino $G$ et al. Pleural plaques: Dose response relationship and clinical significance.

Bignon J. Comments of the difficulty of early diagnosis of mesothelioma.

Tanaka M. Early diagnosis of pneumoconiosis by an ultra-thin bronchofibrescope.
1 Hughes J, Weill $\mathrm{H}$. Asbestos as a precursor of asbestos related lung cancer: results of a prospective mortality study. $\mathrm{Br} \mathcal{F}$ Ind Med 1991;48:229-33.

2 Liddell FDK, McDonald JC. Radiological findings as predictions of mortality in Quebec asbestos workers. $\mathrm{Br} \mathcal{F}$ Ind Med 1980;37:257-67.

3 Jaurand MC, Bignon J, eds. Mesothelial cells and mesothelioma: past, present, and future INSERM Colloque, Paris, 29 September-1 October 1991; to be published in the European Respiratory Journal.

4 McDonald JC, Case BW, Enterline PE, Henderson V, McDonald AD, Plourde M, Sebastien P. Lung dust analysis in the assessment of past exposure of man made mineral fibre workers. Ann Occup Hyg 1990;34:427-441.

5 Sébastien P, Janson X, Gaudichet A, Hirsch A, Bignon J. Asbestos retention in human respiratory tissues: comparative measurements in lung parenchyma and in parietal pleura. In: Wagner JC, ed. Biological effects of mineral fibres. IARC Scientific publication \#30, Vol 1, Lyon: International Agency for Research on Cancer; 237-46. (IARC sci publ No $30 \mathrm{Vol} 1$ ).

6 Case BW, Kuhar M, Harrigan M, Dufresne A. Lung fibre content of American children aged 8-15: preliminary findings. Paper presented at Seventh International Symposium on Inhaled Particles, Edinburgh, UK, September 1991, Proceedings to be published in the Annals of Occupational Hygiene after review and revision.

Accepted 11 January 1993 